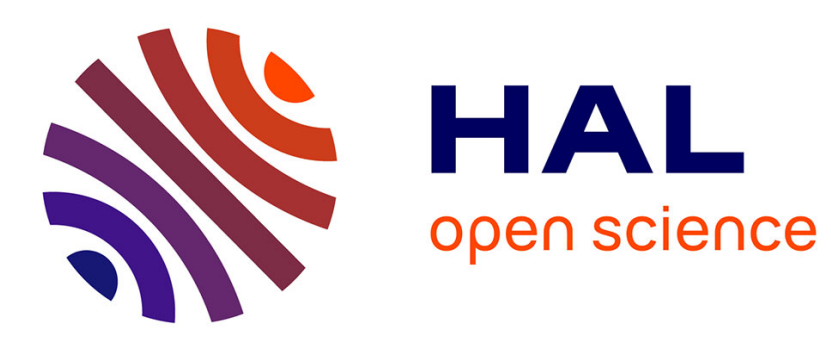

\title{
A new lower bound on the number of perfect matchings in cubic graphs
}

Daniel Král', Jean-Sébastien Sereni, Michael Stiebitz

\section{To cite this version:}

Daniel Král', Jean-Sébastien Sereni, Michael Stiebitz. A new lower bound on the number of perfect matchings in cubic graphs. SIAM Journal on Discrete Mathematics, 2009, 23 (3), pp.1465-1483. $10.1137 / 080723843$. hal-00487173

\section{HAL Id: hal-00487173 \\ https://hal.science/hal-00487173}

Submitted on 28 May 2010

HAL is a multi-disciplinary open access archive for the deposit and dissemination of scientific research documents, whether they are published or not. The documents may come from teaching and research institutions in France or abroad, or from public or private research centers.
L'archive ouverte pluridisciplinaire HAL, est destinée au dépôt et à la diffusion de documents scientifiques de niveau recherche, publiés ou non, émanant des établissements d'enseignement et de recherche français ou étrangers, des laboratoires publics ou privés. 


\title{
A new lower bound on the number of perfect matchings in cubic graphs
}

\author{
Daniel Král ${ }^{*} \quad$ Jean-Sébastien Sereni ${ }^{\dagger} \quad$ Michael Stiebitz $^{\ddagger}$
}

\begin{abstract}
We prove that every $n$-vertex cubic bridgeless graph has at least $n / 2$ perfect matchings and give a list of all 17 such graphs that have less than $n / 2+2$ perfect matchings.
\end{abstract}

\section{Introduction}

Graphs considered in this paper can contain multiple edges but do not contain loops. A graph is cubic if every vertex has degree 3 and a subgraph is spanning if it contains all the vertices. A perfect matching is a spanning subgraph where every vertex has degree 1. A graph is bridgeless if it is connected and stays connected after removing any edge. Let us state the following classical theorem of Petersen [12].

Theorem 1 (Petersen, 1891). Every cubic bridgeless graph $G$ has a perfect matching.

*Institute for Theoretical Computer Science, Faculty of Mathematics and Physics, Charles University, Malostranské náměstí 25, 11800 Prague, Czech Republic. E-mail: kral@kam.mff.cuni.cz. Institute for Theoretical Computer Science (ITI) is supported by the Ministry of Education of the Czech Republic as project 1M0545. This research was also partially supported by the grant GACR 201/09/0197.

${ }^{\dagger}$ CNRS (LIAFA, Université Denis Diderot), Paris, France, and Department of Applied Mathematics (KAM), Faculty of Mathematics and Physics, Charles University, Prague, Czech Republic. E-mail: sereni@kam.mff.cuni.cz. This author's work was supported by the European project IST FET AEOLUS.

${ }^{\ddagger}$ Technische Universität Ilmenau, Institute of Mathematics, P.O.B. 100 565, D-98684 Ilmenau, Germany. E-mail: Michael.Stiebitz@tu-ilmenau.de. 
In fact, a stronger statement is true, as was shown by Plesník [13].

Theorem 2 (Plesník, 1972). Let $G$ be a bridgeless cubic graph. Every edge of $G$ is contained in a perfect matching. Moreover, for any two edges e and $f$ of $G$, there is a perfect matching avoiding both $e$ and $f$.

As a consequence, every cubic bridgeless graph has at least three perfect matchings. A natural question is what is the least number of perfect matchings that an $n$-vertex cubic bridgeless graph contains. Lovász and Plummer conjectured in the mid-1970s that this number grows exponentially with the number of vertices (see the book by Lovász and Plummer $[9$, Conjecture $8.1 .8])$.

Conjecture 1 (Lovász and Plummer, 1970s). Every cubic bridgeless graph with $n$ vertices has at least $2^{\Omega(n)}$ perfect matchings.

Edmonds, Lovász, and Pulleyblank [3, Theorem 5.1] proved the following theorem, which generalizes a result of Naddef [11] for matching covered bipartite graphs (see Section 2 for the relevant definitions).

Theorem 3 (Edmonds, Lovász, and Pulleyblank, 1982). The dimension of the perfect matching polytope of a connected and matching covered graph $G=(V, E)$ is

$$
|E|-|V|+1-b(G),
$$

where $b(G)$ is the number of bricks in the brick and brace decomposition of $G$.

This theorem implies that the dimension of the perfect matching polytope of a cubic bridgeless $n$-vertex graph is at least $n / 4+1$. Since the vertices of the polytope correspond to distinct perfect matchings, we have the following lower bound on the number of perfect matchings of an $n$-vertex cubic bridgeless graph.

Theorem 4 (Edmonds, Lovász, and Pulleyblank, 1982). Every cubic bridgeless graph with $n$ vertices has at least $n / 4+2$ perfect matchings.

If a cubic graph $G$ has no non-trivial edge-cut of size 3, then Theorem 3 gives a better bound, since $G$ must be a brick. A graph $G$ is cyclically $k$ edge-connected if it has no edge-cut of size at most $k-1$ the removal of which yields at least two non-acyclic components. The following result is a simple consequence of Theorem 3. 
Theorem 5 (Edmonds, Lovász, and Pulleyblank, 1982). Every cubic cyclically 4-edge-connected graph with $n$ vertices has at least $n / 2+1$ perfect matchings.

Conjecture 1 has been verified for several special classes of graphs, one of them being bipartite graphs. The first non-trivial lower bound on the number of perfect matchings in cubic bridgeless bipartite graphs was obtained in 1969 by Sinkhorn [17] who proved a bound of $\frac{n}{2}$, thereby establishing a conjecture of Marshall. The same year, Minc [10] increased this lower bound by 2 . Then, a bound of $\frac{3 n}{2}-3$ was proved by Hartfiel and Crosby [7]. The first exponential bound, $6 \cdot\left(\frac{4}{3}\right)^{n / 2-3}$, was obtained in 1979 by Voorhoeve [18]. This was generalized to all regular bipartite graphs in 1998 by Schrijver [14], who thereby proved a conjecture of himself and Valiant [16]. His argument is involved, and we note that, as a particular case of a different and more general approach (using hyperbolic polynomials), Gurvits [6] managed to slightly improve the bound, as well as simplify the proof.

Recently, an important step towards a proof of Conjecture 1 has been made by Chudnovsky and Seymour [2] who proved the conjecture for planar graphs.

Theorem 6 (Chudnovsky and Seymour, 2008). Every cubic bridgeless planar graph with $n$ vertices has at least $2^{n / 655978752}$ perfect matchings.

In this paper, we focus on proving a bound corresponding to that stated in Theorem 5 for all cubic bridgeless graphs, i.e., we remove the assumption that $G$ is cyclically 4-edge-connected. In particular, we prove that every $n$-vertex cubic bridgeless graph $G$ has at least $n / 2$ perfect matchings and provide complete lists of such graphs with exactly $n / 2$ and $n / 2+1$ perfect matchings. Our main result is the following theorem.

Theorem 7. Let $G$ be a cubic bridgeless graph with $n$ vertices. The graph $G$ contains at least $n / 2+2$ perfect matchings unless it is is one of the 17 exceptional graphs $I_{1}, \ldots, I_{10}$ or $H_{0}, \ldots, H_{6}$ which are depicted in Figures 2 , 3 , 4 and 6 . The graph $H_{0}$ contains $n / 2$ perfect matchings and the other exceptional graphs contain $n / 2+1$ perfect matchings.

Using our results, Esperet et al. [5] showed that every cubic bridgeless $n$-vertex graph has at least $3 n / 4-10$ perfect matchings and a superlinear bound has later been found [4]. 


\section{Brick and brace decomposition}

The brick and brace decomposition is one of the essential notions in the theory of perfect matchings. We explain the notion in general though we apply it only to cubic bridgeless graphs. We refer to the treatise of Schrijver [15, Chapter 37] for further exposition. Given a graph $G$ and a subset $X$ of vertices, $G-X$ is the subgraph obtained from $G$ by removing the vertices of $X$. A graph $G$ is matching covered if every edge of $G$ is contained in a perfect matching. If $V_{1}$ and $V_{2}$ is a partition of a vertex set of $G$, then the edges with one end-vertex in $V_{1}$ and the other in $V_{2}$ form an edge-cut. An edge-cut is non-trivial if both $V_{1}$ and $V_{2}$ contain at least two vertices. An edge-cut $E$ is tight if every perfect matching contains exactly one edge of $E$.

Let $G$ be a matching covered graph with a non-trivial tight edge-cut $E$, which partitions the vertices of $G$ into two sets $V_{1}$ and $V_{2}$. We decompose $G$ into two simpler graphs $G_{1}$ and $G_{2}$ by splitting along $E$ as follows: the graph $G_{i}$ is obtained by contracting all the vertices of $V_{i}$ to a single vertex, for $i \in\{1,2\}$. Note that the structure of perfect matchings of $G_{1}$ and $G_{2}$ reflects the structure of perfect matchings of $G$ : no matchings are lost by the splitting since every perfect matching uses exactly one edge of $E$. In particular, the graphs $G_{1}$ and $G_{2}$ are matching covered. If one or both of the new graphs contain a non-trivial tight edge-cut, we can again split along it. We continue until we obtain a multiset of graphs with no non-trivial tight edge-cuts. In doing so, we keep parallel edges that arise (which deviates from some other literature), since multiple edges play an important role regarding the number of perfect matchings. The following theorem of Lovász [8] states that splitting along non-trivial tight edge-cuts is independent of the order in that the edge-cuts were chosen.

Theorem 8 (Lovász, 1987). Let $G$ be a matching covered graph. Up to multiplicities of edges, the multiset of graphs with no non-trivial tight edge-cuts obtained by splitting along non-trivial tight edge-cuts of $G$ depends neither on the chosen edge-cuts nor on the order in which the splittings are performed.

The graphs in the multiset obtained by splitting along non-trivial tight edge-cuts are of two kinds. Bipartite graphs with no non-trivial tight edge-cut are referred to as braces. They are characterized by the following property [3].

Theorem 9 (Edmonds, Lovász, and Pulleybank, 1982). A bipartite matching covered graph $G$ has no non-trivial tight edge-cut if and only if for every pair 


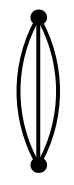

$A_{1}$

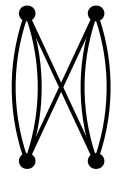

$A_{2}$

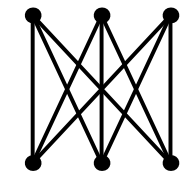

$A_{3}$

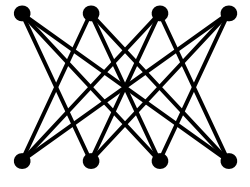

$A_{4}$

Figure 1: Cubic braces of order at most 4 .

of subsets $V$ and $W$ from different color classes such that $|V|=|W| \leq 2$, the graph $G-(V \cup W)$ has a perfect matching.

If $G$ is a cubic bridgeless graph that is a brace, shortly a cubic brace, we call the number of vertices in each color class of $G$ the order of the brace. There is a unique cubic brace $A_{n}$ of order $n$ for each $n \in\{1,2,3,4\}$. The braces $A_{1}, \ldots, A_{4}$ can be found in Figure 1.

Non-bipartite graphs that appear in the decomposition of a matching covered graph along its non-trivial tight edge-cuts are known as bricks. They are characterized as follows [3].

Theorem 10 (Edmonds, Lovász, and Pulleybank, 1982). A non-bipartite matching covered graph $G$ has no non-trivial tight edge-cut if and only if it is 3-connected and for every two-element subset $V$ of its vertices, the graph $G-V$ has a perfect matching.

As in the case of braces, we refer to bricks that are cubic bridgeless graphs as to cubic bricks. Examples of cubic bricks can be found in Figure 2. Since the decomposition of a graph $G$ along its non-trivial tight edge-cuts is formed by bricks and braces, it is called the brick and brace decomposition of $G$. Recall that this decomposition is unique by Theorem 8 . The brick and brace decomposition is non-trivial if it contains at least two graphs, i.e., the brick and brace decomposition of $G$ is non-trivial if and only if $G$ is neither a brick nor a brace.

In the rest of this section, we deal with cubic bridgeless graphs only. Before our further considerations, let us state the following consequence of the structure of the perfect matching polytope of a cubic bridgeless graph $G$ : every tight edge-cut of $G$ has size 3. Indeed, if $E$ is a tight edge-cut of $G$, then for every vector corresponding to a perfect matching of $G$, the coordinates of the edges of $E$ sum-up to exactly 1 . Thus, the same is true for 
every vector of the perfect matching polytope of $G$. The conclusion follows by observing that the vector with all coordinates equal to $1 / 3$ belongs to the perfect matching polytope of $G$ (which is a direct consequence of Edmonds' description of the perfect matching polytope of a graph). As a result, the graphs forming the brick and brace decomposition of a cubic bridgeless graph are also cubic and bridgeless. Furthermore, it follows from Theorem 10 that every cubic brick is a simple graph.

We now state and prove some basic lemmas on the brick and brace decompositions of cubic bridgeless graphs, and on cubic bricks and cubic braces. Though the reader can be familiar with some of these facts, we give their short proofs for completeness. Before our first lemma, we need two more definitions. A vertex $v$ of a cubic graph $G$ is trimatched if there exists a spanning subgraph $H$ of $G$ such that the degree of $v$ in $H$ is 3 and the degrees of the other vertices of $H$ are 1 . The graph $G$ is trimatched if every vertex of $G$ is trimatched. If $G$ is a simple graph, then a vertex $v$ with neighbors $v_{1}, v_{2}$ and $v_{3}$ is trimatched if and only if the graph $G-\left\{v, v_{1}, v_{2}, v_{3}\right\}$ has a perfect matching.

Lemma 11. Every cubic brick $G$ is trimatched.

Proof. Let $v$ be any vertex of $G$ and $v_{1}, v_{2}$ and $v_{3}$ its neighbors. By Theorem 10 , the graph $G-\left\{v_{2}, v_{3}\right\}$ has a perfect matching $M$. Since $G$ is cubic, this perfect matching includes the edge $v v_{1}$. Since every cubic brick is simple, the perfect matching $M$ together with the edges $v v_{2}$ and $v v_{3}$ is a spanning subgraph of $G$ of the type sought.

Using Lemma 11, we show that every non-trivial brick and brace decomposition contains a brace.

Lemma 12. Every non-trivial brick and brace decomposition of a cubic bridgeless graph contains a brace.

Proof. It is enough to prove that there is no graph with a brick and brace decomposition consisting of two bricks. Suppose on the contrary that $G$ is such a graph. Let $E:=\left\{v_{1} w_{1}, v_{2} w_{2}, v_{3} w_{3}\right\}$ be a non-trivial tight edge-cut of $G$ : it partitions the vertex-set of $G$ into two sets $V_{1}$ and $V_{2}$. Let $G_{1}$ and $G_{2}$ be the two bricks obtained by splitting along $E$. We may assume that $G_{1}$ contains the vertices $v_{i}$ and we let $u_{1}$ be the vertex of $G_{1}$ obtained by contracting the vertices of $V_{2}$. Similarly, $G_{2}$ contains the vertices $w_{i}$ and we let $u_{2}$ be the vertex of $G_{2}$ obtained by contracting the vertices of $V_{1}$. 
By Lemma 11, both bricks $G_{1}$ and $G_{2}$ are trimatched. In particular, for $i \in\{1,2\}$, the vertex $u_{i}$ is trimatched in $G_{i}$. Let $H_{i}$ be a spanning subgraph of $G_{i}$ such that $u_{i}$ has degree 3 in $H_{i}$ and the other vertices have degree 1 . The subgraphs $H_{1}$ and $H_{2}$ combine to a perfect matching of $G$ including all three edges of $E$, which contradicts our assumption that $E$ is tight.

Let us now turn our attention to cubic braces. Again, we have to introduce a definition. An edge of a matching covered graph $G$ is a solo-edge if it is contained in exactly one perfect matching. A matching covered graph is matching double-covered if it has no solo-edges.

Lemma 13. Every cubic brace different from $A_{1}$ and $A_{2}$ (i.e. every cubic brace of order at least 3) is matching double-covered.

Proof. Let $G$ be a cubic brace. Since $A_{1}$ and $A_{2}$ are the only cubic braces of order at most 2, the order of the brace $G$ is at least 3 . Let $u v$ be an edge of $G$ and $M$ a matching containing $u v$. Since the order of $G$ is greater than 2, there exists an edge $u^{\prime} v^{\prime}$ not in $M$ and not adjacent with $u v$. By Theorem 9, the graph $G-\left\{u, v, u^{\prime}, v^{\prime}\right\}$ has a perfect matching $M^{\prime}$. We can extend $M^{\prime}$ to $G$ by adding the edges $u v$ and $u^{\prime} v^{\prime}$. Thus, $M$ and $M^{\prime}$ are two distinct perfect matchings of $G$ containing the edge $u v$. Consequently, $G$ has no solo-edge.

We finish this section with a lemma on cubic graphs the decomposition of which contains a brace different from $A_{1}$ and $A_{2}$.

Lemma 14. Every cubic bridgeless graph $G$ with a brick and brace decomposition containing a brace different from $A_{1}$ and $A_{2}$ is matching double-covered.

Proof. We proceed by induction on the number $k$ of graphs in the brick and brace decomposition of $G$. If $k=1$, then $G$ is matching double-covered by Lemma 13. Assume that $k \geq 2$ and let us show that $G$ is matching doublecovered. To this end, let $e$ be an edge of $G$. Consider any non-trivial tight edge-cut $E$ of $G$. Let $G_{1}$ and $G_{2}$ be the graphs obtained from $G$ by splitting along this edge-cut.

By Theorem 8, the brick and brace decomposition of $G_{1}$ or $G_{2}$ contains a brace different from $A_{1}$ and $A_{2}$. Assume that $G_{1}$ has this property. Thus, $G_{1}$ is matching double-covered by induction.

If $e$ is in $G_{1}$, then $G_{1}$ contains two distinct perfect matchings containing $e$, and each of them can be extended to a perfect matching of $G$ since $G_{2}$ is matching covered. Hence, $e$ is not a solo-edge. 
If $e$ is in $G_{2}$, then a perfect matching of $G_{2}$ containing $e$ can be extended to a perfect matching of $G$ in at least two different ways, since $G_{1}$ is matching double-covered. Consequently, $e$ is not a solo-edge either.

\section{Good cubic graphs}

In this section, we present most of our tools for proving the lower bounds of Theorem 7 on the number of perfect matchings in a cubic bridgeless graph. Let us start with some terminology. An $n$-vertex cubic bridgeless graph $G$ is $\alpha$-good if $G$ has $n / 2+\alpha$ perfect matchings, and $G$ is $(\geq \alpha)$-good if it has at least $n / 2+\alpha$ perfect matchings. By Theorem 3 , the dimension of the perfect matching polytope of an $n$-vertex cubic brick is $\frac{n}{2}$. A theorem of de Carvalho, Lucchesi, and Murty [1] charaterizes cubic bricks that have a simplex with $\frac{n}{2}+1$ vertices as the perfect matching polytope. Their result implies that every brick is $(\geq 2)$-good except the bricks $I_{1}, \ldots, I_{10}$ depicted in Figure 2.

Theorem 15 (de Carvalho, Lucchesi, and Murty, 2005). Every brick different from the 10 bricks $I_{1}, \ldots, I_{10}$ depicted in Figure 2 is $(\geq 2)$-good. All the bricks $I_{1}, \ldots, I_{10}$ are 1-good.

Our lower bound argument is based on the analysis of the brick and brace decompositions of cubic bridgeless graphs. We have introduced the operation of splitting along non-trivial tight edge-cuts in Section 2. We now define the inverse operation. Let $G_{1}$ and $G_{2}$ be cubic bridgeless graphs, $u$ a vertex of $G_{1}$ with neighbors $u_{1}, u_{2}$ and $u_{3}$, and $v$ a vertex of $G_{2}$ with neighbors $v_{1}$, $v_{2}$ and $v_{3}$. Let $G$ be the graph obtained from $G_{1}$ and $G_{2}$ by removing the vertices $u$ and $v$ and adding the edges $u_{1} v_{1}, u_{2} v_{2}$ and $u_{3} v_{3}$. We say that $G$ is obtained by gluing the graphs $G_{1}$ and $G_{2}$, or more precisely from $G_{1}$ by gluing $G_{2}$ through the vertex $u$, or from $G_{2}$ by gluing $G_{1}$ through the vertex $v$. The gluing is a solo-gluing if for every $i \in\{1,2,3\}$, the edge $u u_{i}$ is a solo-edge in $G_{1}$ or the edge $v v_{i}$ is a solo-edge in $G_{2}$.

We now prove two lemmas giving lower bounds on the number of perfect matchings in graphs obtained by gluing smaller graphs. Before doing so, let us introduce one more definition. If $G$ is a cubic bridgeless graph and $v$ a vertex of $G$ with neighbors $v_{1}, v_{2}$ and $v_{3}$, then the pattern of $v$ is the triple $\left(m_{1}, m_{2}, m_{3}\right)$ where $m_{i}$ is the number of perfect matchings of $G$ containing the edge $v v_{i}$ for $i \in\{1,2,3\}$. We are now ready to prove the two lemmas. 


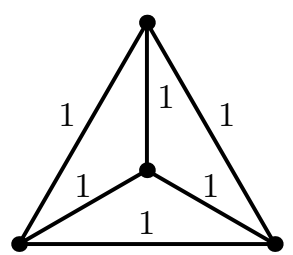

$I_{1}$

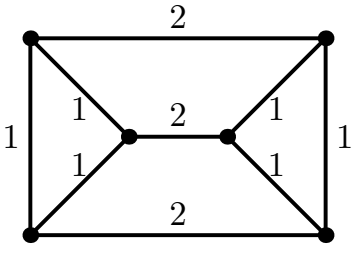

$I_{2}$

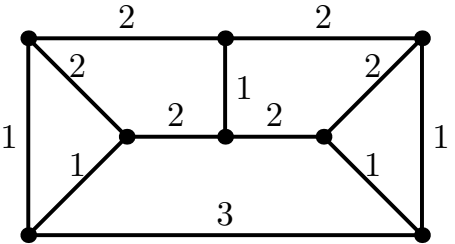

$I_{3}$
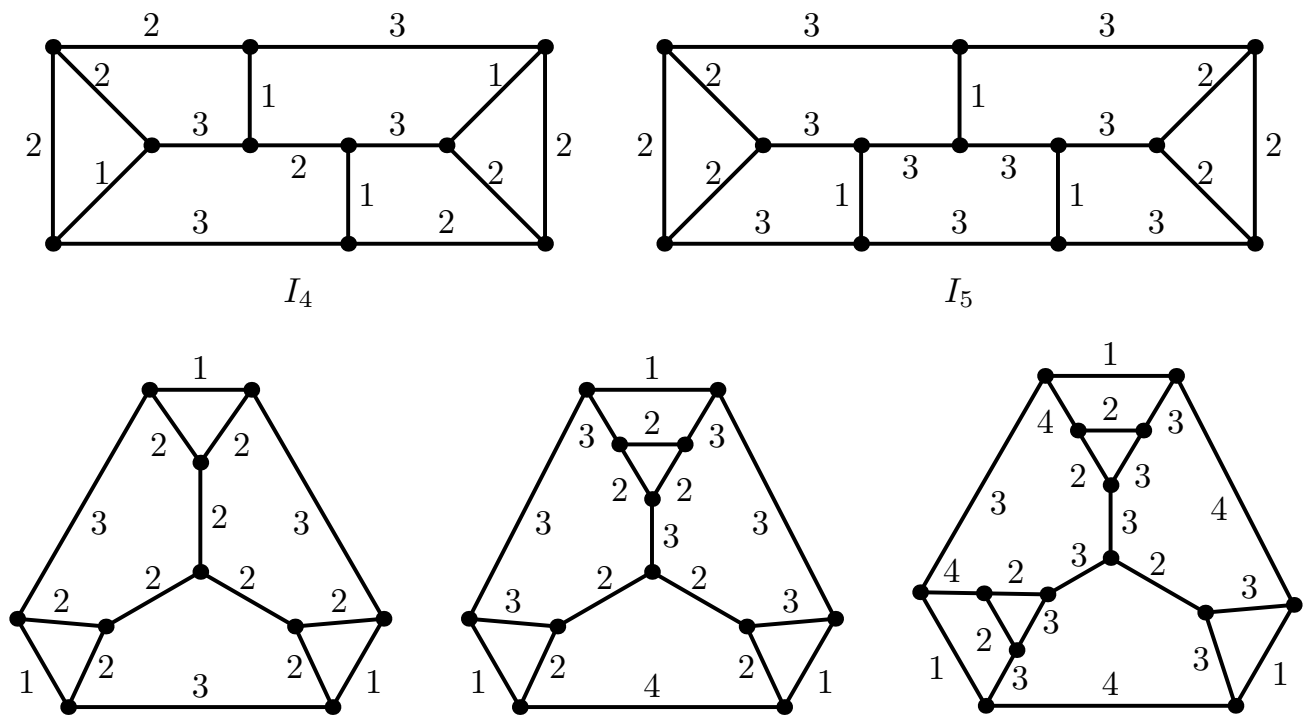

$I_{6}$

$I_{7}$

$I_{8}$
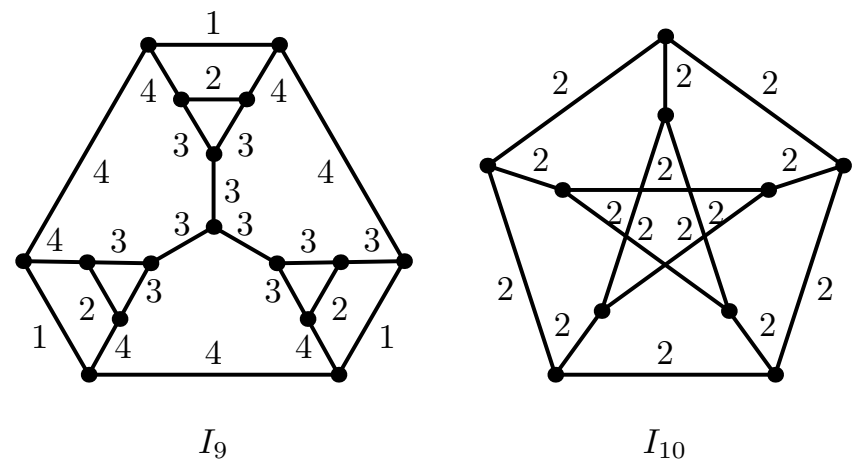

Figure 2: All 1-good bricks. The number near each edge indicates the number of perfect matchings containing this edge. 
Lemma 16. Let $G$ be a cubic bridgeless graph obtained by gluing an $\alpha$-good graph $G_{a}$ and a $\beta$-good graph $G_{b}$. The graph $G$ is $(\geq \alpha+\beta-1)$-good unless $G$ is obtained by a solo-gluing, in which case $G$ is $(\alpha+\beta-2)$-good.

Proof. Let $n_{a}$ be the number of vertices of $G_{a}$ and $n_{b}$ the number of vertices of $G_{b}$. Next, let $v_{a}$ be the vertex of $G_{a}$ such that $G$ is obtained from $G_{a}$ by gluing $G_{b}$ through $v_{a}$. Similarly, $v_{b}$ is the vertex of $G_{b}$ such that $G$ is obtained from $G_{b}$ by gluing $G_{a}$ through $v_{b}$. Finally, let $\left(m_{a, 1}, m_{a, 2}, m_{a, 3}\right)$ be the pattern of $v_{a}$ in $G_{a}$ and $\left(m_{b, 1}, m_{b, 2}, m_{b, 3}\right)$ the pattern of $v_{b}$ in $G_{b}$.

Since $G_{a}$ is $\alpha$-good and $G_{b}$ is $\beta$-good,

$$
n_{a} / 2+\alpha=m_{a, 1}+m_{a, 2}+m_{a, 3}
$$

and

$$
n_{b} / 2+\beta=m_{b, 1}+m_{b, 2}+m_{b, 3} .
$$

Observe that $x y \geq x+y-1$ for every positive integers $x$ and $y$, with equality if and only $x=1$ or $y=1$. Hence, the definition of gluing and the fact that $m_{a, i} \geq 1$ and $m_{b, i} \geq 1$ yield that the number of perfect matchings of $G$ is at least

$$
m_{a, 1} m_{b, 1}+m_{a, 2} m_{b, 2}+m_{a, 3} m_{b, 3} \geq m_{a, 1}+m_{a, 2}+m_{a, 3}+m_{b, 1}+m_{b, 2}+m_{b, 3}-3
$$

with equality if and only if for every $i \in\{1,2,3\}$, at least one of the numbers $m_{a, i}$ and $m_{b, i}$ equals 1 . Since $G$ has $n_{a}+n_{b}-2$ vertices, (1), (2) and (3) imply that $G$ is $(\geq \alpha+\beta-2)$-good. Moreover, $G$ is $(\geq \alpha+\beta-1)$-good unless at least one of the numbers $m_{a, i}$ and $m_{b, i}$ equals 1 for every $i \in\{1,2,3\}$, i.e. unless $G$ is obtained by a solo-gluing.

In the final lemma of this section, we show that the bound from Lemma 16 can be improved if one of the glued graphs is matching double-covered.

Lemma 17. Let $G$ be a cubic bridgeless graph obtained by gluing an $\alpha$-good graph $G_{a}$ and a $\beta$-good graph $G_{b}$. If $G_{a}$ is matching double-covered and $G_{b}$ has at least five perfect matchings, then $G$ is $(\alpha+\beta)$-good.

Proof. Let us retain the notation from the proof of Lemma 16. Assume that $m_{b, 1} \geq m_{b, 2} \geq m_{b, 3}$, and let $p$ be the number of perfect matchings of $G$. It is still true that

$$
p=m_{a, 1} m_{b, 1}+m_{a, 2} m_{b, 2}+m_{a, 3} m_{b, 3} .
$$


First, assume that $m_{b, 2}=m_{b, 3}=1$. Hence, $m_{b, 1} \geq 3$ since $G_{b}$ has at least five perfect matchings. Note that $m_{a, i} \geq 2$ for every $i \in\{1,2,3\}$ since $G_{a}$ is matching double-covered. In particular, $m_{a, 1} m_{b, 1} \geq m_{a, 1}+m_{b, 1}+1$ since $x y \geq x+y+1$ for any $x \geq 2$ and $y \geq 3$. Thus, (4) translates to

$$
\begin{aligned}
p & \geq m_{a, 1}+m_{b, 1}+1+m_{a, 2}+m_{a, 3} \\
& =m_{a, 1}+m_{a, 2}+m_{a, 3}+m_{b, 1}+m_{b, 2}+m_{b, 3}-1 \\
& =\frac{n_{a}}{2}+\frac{n_{b}}{2}+\alpha+\beta-1,
\end{aligned}
$$

by the equations (1) and (2). Since the number of vertices of $G$ is $n_{a}+n_{b}-2$, we deduce that $G$ is $(\alpha+\beta)$-good.

We next assume that both $m_{b, 1}$ and $m_{b, 2}$ are at least 2. Again recalling that $x y \geq x+y-1$ for two positive integers $x$ and $y$, with equality if and only if $x=1$ or $y=1$, we deduce from (4) that

$$
p \geq m_{a, 1}+m_{b, 1}+m_{a, 2}+m_{b, 2}+m_{a, 3}+m_{b, 3}-1 \geq \frac{n_{a}}{2}+\frac{n_{b}}{2}-1 .
$$

Therefore, $G$ is $(\alpha+\beta)$-good.

\section{Bipartite cubic graphs}

In this section, we revisit a simple bound on the number of perfect matchings in bipartite graphs, which can be found in the book of Lovász and Plummer [9]. We need to slightly tune up the constants of the original bound so that they are good enough for our later considerations. Let us start by defining two auxiliary functions $f, g: \mathbb{N} \rightarrow \mathbb{N}$ recursively, as follows.

$$
\begin{aligned}
& g(n)= \begin{cases}2 & \text { if } n=1, \\
\left\lceil\frac{4}{3} g(n-1)\right\rceil & \text { otherwise, }\end{cases} \\
& f(n)=\left\lceil\frac{3}{2} g(n)\right\rceil \text { for every } n \geq 1 .
\end{aligned}
$$

The values of the functions $f(n)$ and $g(n)$ for small $n$ can be found in Table 1 .

We follow the lines of the proof of Theorem 8.1.7 from the book of Lovász and Plummer [9, Chapter 8] to prove the next lemma. In our further considerations, a bipartite graph is near-cubic if all its vertices have degree 3 except one vertex in each color class that has degree 2. 


\begin{tabular}{|c|cccccccccc|}
\hline$n$ & 1 & 2 & 3 & 4 & 5 & 6 & 7 & 8 & 9 & 10 \\
\hline$g(n)$ & 2 & 3 & 4 & 6 & 8 & 11 & 15 & 20 & 27 & 36 \\
\hline$f(n)$ & 3 & 5 & 6 & 9 & 12 & 17 & 23 & 30 & 41 & 54 \\
\hline
\end{tabular}

Table 1: The values $f(n)$ and $g(n)$ for $n \in\{1, \ldots, 10\}$.

Lemma 18. For each positive integer $n$, every cubic bipartite $2 n$-vertex graph contains at least $f(n)$ perfect matchings and every near-cubic bipartite $2 n$ vertex graph contains at least $g(n)$ perfect matchings.

Proof. The proof proceeds by induction on $n$. The only cubic bipartite 2vertex graph is the brace $A_{1}$, which has $3=f(1)$ perfect matchings. The only near-cubic bipartite 2 -vertex graph is obtained from $A_{1}$ by removing an edge: it has $2=g(1)$ perfect matchings. Thus, the bounds claimed in the statement of the lemma hold if $n=1$.

Assume that $n \geq 2$. Let us first consider a near-cubic bipartite $2 n$-vertex graph $G$ and let $u$ and $v$ be its vertices of degree 2. If $u$ and $v$ are adjacent, we show that $G$ contains at least $f(n-1)$ perfect matchings. Indeed, let $v^{\prime}$ be the neighbor of $u$ distinct from $v$ and $u^{\prime}$ the neighbor of $v$ distinct from $u$. Let $G^{\prime}$ be the graph obtained from $G$ by removing the vertices $u$ and $v$, and adding an edge between $u^{\prime}$ and $v^{\prime}$. Since $G^{\prime}$ is a cubic bipartite graph, it contains at least $f(n-1)$ perfect matchings by the induction hypothesis. The perfect matchings of $G^{\prime}$ that contain the edge $u^{\prime} v^{\prime}$ can be converted to perfect matchings of $G$ by replacing the edge $u^{\prime} v^{\prime}$ with the edges $u v^{\prime}$ and $u^{\prime} v$, and those matchings of $G^{\prime}$ that avoid the edge $u^{\prime} v^{\prime}$ can be extended to perfect matchings of $G$ by adding the edge $u v$. Since different perfect matchings of $G^{\prime}$ yield different perfect matchings of $G$, we deduce that $G$ has at least $f(n-1)$ perfect matchings. The desired bound follows since $f(n-1) \geq \frac{4}{3} g(n-1)$.

We now consider the case where the vertices $u$ and $v$ are not adjacent. First, if $u$ has only one neighbor $v^{\prime}$ (so there are two parallel edges between $u$ and $v^{\prime}$ ), then let $G^{\prime}$ be the subgraph of $G$ obtained by removing $u$ and $v^{\prime}$. Since $G^{\prime}$ is a near-cubic bipartite graph, the induction hypothesis implies that $G^{\prime}$ has at least $g(n-1)$ perfect matchings. Each of these perfect matchings can be extended to a perfect matching of $G$ in two different ways, by adding one of the two edges between $u$ and $v^{\prime}$. Since different perfect matchings of $G^{\prime}$ yield different perfect matchings of $G$, we deduce that $G$ has at least $2 \cdot g(n-1)$ perfect matchings, which is more than $g(n)$. So assume now that $u$ 
has two distinct neighbors $v_{1}$ and $v_{2}$. For $i \in\{1,2\}$, let $u_{i}$ and $u_{i}^{\prime}$ be the two neighbors of $v_{i}$ different from $u$ (these vertices need not be distinct). Finally, let $G_{1}, G_{2}, G_{3}$ and $G_{4}$ be the four graphs obtained from $G$ by removing the vertex $u$, removing one of the four edges $u_{1} v_{1}, u_{1}^{\prime} v_{1}, u_{2} v_{2}$ and $u_{2}^{\prime} v_{2}$, and identifying the vertices $v_{1}$ and $v_{2}$. Each of the four graphs $G_{i}$ is a near-cubic bipartite graph.

Every perfect matching of $G_{i}$ corresponds to a perfect matching of $G$, e.g., any perfect matching of $G_{1}$ can be completed to a perfect matching of $G$ by adding the edge $u v_{1}$ or $u v_{2}$. On the other hand, a perfect matching of $G$ corresponds to perfect matchings in exactly three of the graphs $G_{1}, \ldots, G_{4}$ since it includes exactly one of the four edges $u_{1} v_{1}, u_{1}^{\prime} v_{1}, u_{2} v_{2}$ and $u_{2}^{\prime} v_{2}$. Hence, $G$ has at least $4 g(n-1) / 3$ perfect matchings.

We have shown that $G$ contains at least $4 g(n-1) / 3$ perfect matchings. Since the number of perfect matchings of $G$ is an integer, $G$ contains at least $g(n)$ perfect matchings, as asserted.

Assume now that $H$ is a bipartite cubic graph. Let $v$ be a vertex of $H$ and $v_{1}, v_{2}$ and $v_{3}$ the three (non-necessarily all distinct) neighbors of $v$. For $i \in\{1,2,3\}$, let $H_{i}$ be the near-cubic bipartite graph obtained by removing the edge $v v_{i}$. As shown before, $H_{i}$ contains at least $g(n)$ perfect matchings. If $M$ is a perfect matching of $H$, then $M$ is also a perfect matching of exactly two of the graphs $H_{1}, H_{2}$ and $H_{3}$. Hence, $H$ contains at least $3 g(n) / 2$ perfect matchings. Since the number of perfect matchings is an integer, $H$ contains at least $f(n)=\lceil 3 g(n) / 2\rceil$ perfect matchings.

Lemma 19. For each $n \geq 5$, every brace $G$ of order $n$ is $(\geq n+2)$-good.

Proof. Since $g(5)=8$, we infer that for all $n \geq 5$,

$$
f(n) \geq \frac{3}{2} \cdot\left(\frac{4}{3}\right)^{n-5} \cdot 8=\frac{4^{n-4}}{3^{n-6}} \geq 2 n+2 .
$$

By Lemma 18, $G$ has at least $f(n) \geq 2 n+2$ perfect matchings and thus $G$ is $(\geq n+2)$-good.

We finish this section by obtaining a simple constant lower bound on the number of perfect matchings in cubic bridgeless graphs, which turns out to be useful in our further considerations.

Lemma 20. Every cubic bridgeless graph different from $A_{1}, I_{1}$ and $I_{2}$ has at least five perfect matchings. 
Proof. Let $G$ be a cubic bridgeless graph. If $G$ is a brace then, by Lemma 18, the graph $G$ has at least five perfect matchings unless $G=A_{1}$. If $G$ has a non-trivial brick and brace decomposition, then its decomposition contains a brace by Lemma 12, which cannot be $A_{1}$. Hence, the brace in the decomposition of $G$ has at least five perfect matchings. Since the number of perfect matchings of a graph is at least the minimum of the number of perfect matchings of the graphs in its brick and brace decomposition (because every perfect matching of a graph in the decomposition can be extended to a perfect matching of the original graph), $G$ has at least five perfect matchings.

It remains to consider the case where $G$ is a brick. By Theorem 15, every $n$-vertex brick has at least $n / 2+1$ perfect matchings. Hence, if $G$ has less than five perfect matchings, then $G$ has at most six vertices. But the only two bricks with at most six vertices are the bricks $I_{1}$ and $I_{2}$.

\section{$5 \quad$ Single-brace cubic graphs}

In this section, we analyze the number of perfect matchings in graphs the brick and brace decomposition of which contains exactly one brace. Such cubic bridgeless graphs are referred to as single-brace graphs. Before we proceed further, let us state a simple lemma on trimatched vertices in cubic graphs.

Lemma 21. If $G$ is a cubic bridgeless graph obtained from $G^{\prime}$ by gluing a graph $G^{\prime \prime}$ through a vertex $v$, then every vertex $w \neq v$ of $G^{\prime}$ that is trimatched in $G^{\prime}$ is also trimatched in $G$.

Proof. Let $H^{\prime}$ be a spanning subgraph of $G^{\prime}$ such that the vertex $w$ has degree 3 in $H^{\prime}$ and the other vertices of $G^{\prime}$ have degree 1 . Let $e$ be the edge of $H^{\prime}$ incident with $v$ and let $f$ be the edge corresponding to $e$ in $G^{\prime \prime}$. Let $M$ be a perfect matching of $G^{\prime \prime}$ that contains the edge $f$ (recall that every cubic bridgeless graph is matching covered). The subgraph $H^{\prime}$ and the matching $M$ combine to a spanning subgraph $H$ of $G$ where the degree of $w$ is 3 and the degrees of other vertices are 1 . Hence, the vertex $w$ is trimatched in $G$.

Let us now apply Lemma 21 to establish the following auxiliary lemma restricting the set of vertices through which a brick can be glued to a brace. 
Lemma 22. Let $G$ be a single-brace graph. If the brick and brace decomposition consists of a brace $B$ of order $n$ and bricks $B_{1}, \ldots, B_{k}$, and the brace $B$ is not $A_{2}$, then $k \leq n$ and $G$ can be obtained from $B$ by gluing $B_{i}$ through a vertex $v_{i}$ of $B$ for each $i \in\{1, \ldots, k\}$ such that all the vertices $v_{i}$ are in the same color class of $B$.

Proof. The proof proceeds by induction on $k$, the conclusion holding trivially when $k=1$. Assume that $k \geq 2$. Let us consider a non-trivial tight edge-cut $E$ of $G$ and let $G_{1}$ and $G_{2}$ be the two graphs obtained by splitting along the edge-cut $E$. By Theorem 8 and Lemma 12, one of the graphs $G_{1}$ and $G_{2}$ is a brick. By symmetry, we can assume that $G_{2}$ is the brick $B_{k}$. Let $w$ be the vertex such that $G$ is obtained from $G_{1}$ by gluing $B_{k}$ through $w$.

By the induction hypothesis, for each $i \in\{1, \ldots, k-1\}$, the graph $G_{1}$ is obtained from the brace $B$ by gluing $B_{i}$ through a vertex $v_{i}$, and the vertices $v_{1}, \ldots, v_{k-1}$ are in the same color class of $B$. In order to finish the proof of the lemma, we have to exclude the following two cases.

- The vertex $w$ is a vertex of one of the bricks $B_{1}, \ldots, B_{k-1}$.

- The vertex $w$ is not in the color class containing the vertices $v_{1}, \ldots, v_{k-1}$.

To this end, we show that if $w$ is of one of the above two types, then $w$ is trimatched in $G_{1}$. Since $G_{2}$ is trimatched by Lemma 11, this would imply that the edge-cut $E$ is not tight. If $w$ is a vertex of one of the bricks, then it is trimatched by Lemma 21 (where we apply this lemma several times while gluing the bricks to construct $\left.G_{1}\right)$. Hence, we have to focus on the case where $w$ is not in the color class containing the vertices $v_{1}, \ldots, v_{k-1}$.

Since the brace $A_{1}$ does not appear in any non-trivial brick and brace decomposition and $B \neq A_{2}$, the brace $B$ is simple (by Theorem 9). Let $w^{\prime}$ and $w^{\prime \prime}$ be two neighbors of $w$ distinct from $v_{1}$, and let $v^{\prime}$ and $v^{\prime \prime}$ be two neighbors of $v_{1}$ distinct from $w$. By Theorem 9 , the graph $B-\left\{v^{\prime}, v^{\prime \prime}, w^{\prime}, w^{\prime \prime}\right\}$ has a perfect matching. Adding the edges $v_{1} v^{\prime}, v_{1} v^{\prime \prime}, w w^{\prime}$ and $w w^{\prime \prime}$ to this perfect matching yields a spanning subgraph $H_{B}$ of $B$, all the vertices of which have degree 1 except for the vertices $v_{1}$ and $w$, which both have degree 3. Along the brick and brace decomposition, using the fact that the bricks are trimatched by Lemma 11, the subgraph $H_{B}$ can be extended to a spanning subgraph $H$ of $G_{1}$ in which every vertex has degree 1 except the vertex $w$, which has degree 3. Hence, $w$ is trimatched in $G_{1}$.

Since gluing a brick through a trimatched vertex does not create a new non-trivial tight edge-cut, the vertex $w$ must belong to the same color class 


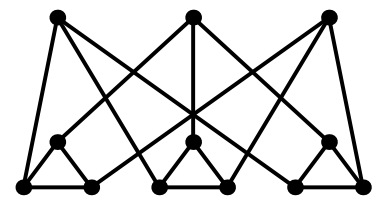

$H_{0}$

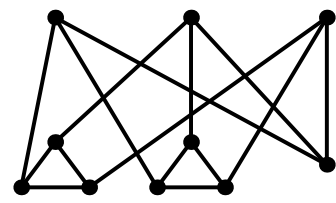

$H_{1}$

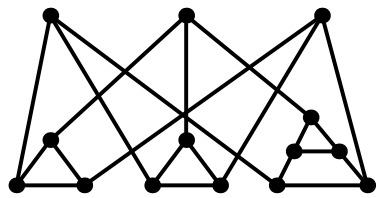

$\mathrm{H}_{2}$

Figure 3: The exceptional graphs $H_{0}, H_{1}$ and $H_{2}$.

as $v_{1}, \ldots, v_{k-1}$. In particular, $G$ can be obtained from the brace $B$ by gluing the bricks $B_{1}, \ldots, B_{k}$ through vertices $v_{1}, \ldots, v_{k}$ contained in the same color class. Since each color class of $B$ contains $n$ vertices, the number $k$ of bricks is at most $n$. The proof of the lemma is now finished.

With Lemma 22, we are ready to consider single-brace graphs the decomposition of which contains the brace $A_{3}$.

Lemma 23. If $G$ is a single-brace graph that contains $A_{3}$ in its brick and brace decomposition, then $G$ is $(\geq 2)$-good unless it is one of the graphs $H_{0}$, $H_{1}$ and $H_{2}$ depicted in Figure 3.

Proof. By Lemma 22, the graph $G$ is obtained from the brace $A_{3}$ by gluing at most three bricks through vertices of the same color class of $A_{3}$. Let $i_{1}$ be the number of bricks $I_{1}$ glued to $A_{3}, i_{2}$ the number of bricks $I_{2}$ glued to $A_{3}$, and $i$ the number of other bricks glued to $A_{3}$. Thus, $i_{1}+i_{2}+i \leq 3$.

The graph $A_{3}$ is 3-good and matching double-covered (the latter being implied by Lemma 13). Since $I_{1}$ is 1 -good, the graph $G_{1}$ obtained by gluing $i_{1}$ bricks $I_{1}$ to $A_{3}$ is $\left(\geq 3-i_{1}\right)$-good by Lemma 16 .

Let $G_{2}$ be the graph obtained from $G_{1}$ by gluing $i_{2}$ bricks $I_{2}$ according to the brick and brace decomposition of $G$. Note that $I_{2}$ is 1 -good, and no vertex of $I_{2}$ is incident with three solo-edges. Moreover, the graph $G_{1}$ is matching double-covered by Lemma 14. Consequently, none of these $i_{2}$ gluings is a solo-gluing. Hence, the graph $G_{2}$ is $\left(\geq 3-i_{1}\right)$-good by Lemma 16 .

Finally, each of the remaining $i$ bricks contains at least five perfect matchings by Lemma 20 and is $(\geq 1)$-good by Theorem 15 . Since the graph $G_{2}$ is matching double-covered by Lemma 14 , the final graph $G$ is $\left(\geq 3-i_{1}+i\right)$ good by Lemma 17 . Hence, if $G$ is not $(\geq 2)$-good, then $i_{1} \geq 2+i$. Since $i_{1}+i_{2}+i \leq 3$, we deduce that $i=0$ and $i_{2} \in\{0,1\}$. So, either $i_{1}=3$ and 


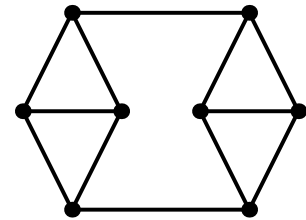

$H_{3}$

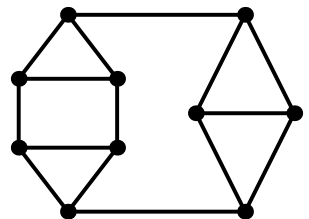

$H_{4}$

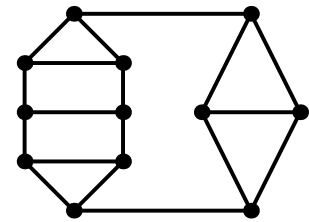

$H_{5}$

Figure 4: The exceptional graphs $H_{3}, H_{4}$ and $H_{5}$.

$i_{2}=0$, or $i_{1}=2$ and $i_{2}=0$, or $i_{1}=2$ and $i_{2}=1$. The graph $G$ is then either $H_{0}, H_{1}$, or $H_{2}$, respectively. It is straightforward to verify that $H_{0}$ is 0 -good and the graphs $H_{1}$ and $H_{2}$ are 1-good.

Before we proceed to analyze single-brace graphs the brick and brace decomposition of which contains a brace of order at least 4 , let us deal with the case where the decomposition contains the brace $A_{2}$.

Lemma 24. If $G$ is a single-brace graph that contains $A_{2}$ in its brick and brace decomposition, then $G$ is $(\geq 2)$-good unless it is one of the graphs $\mathrm{H}_{3}$, $\mathrm{H}_{4}$ and $\mathrm{H}_{5}$ depicted in Figure 4 .

Proof. Let $B=A_{2}$ be the brace and $B_{1}, \ldots, B_{k}$ the bricks forming the brick and brace decomposition of $G$. As in the proof of Lemma 22, it is possible to argue using Lemma 21 that $G$ is obtained by gluing $B_{1}, \ldots, B_{k}$ through distinct vertices $v_{1}, \ldots, v_{k}$ of the brace $B$ (this part of the proof only used the fact that every brick is trimatched). However, since $B$ is not simple, it is not possible to argue that the vertices $v_{1}, \ldots, v_{k}$ are in the same color class of $A_{2}$ as in the proof of Lemma 22. In fact, they do not have to, as we shall see in what follows.

Although the vertices $v_{1}, \ldots, v_{k}$ do not have to be contained in the same color class of $B$, we assert it is still true that $k \leq 2$. Suppose on the contrary that $k \geq 3$. Then, two of the vertices $v_{i}$, say $v_{1}$ and $v_{2}$, are in the same color class of $B$. We show that the graph $G^{\prime}$ obtained from $B$ by gluing the brick $B_{1}$ through the vertex $v_{1}$ and the brick $B_{2}$ through the vertex $v_{2}$ is trimatched. Since $B_{3}$ is a brick, and thus is trimatched by Lemma 11, this will eventually contradict the assumption that the edge-cut of $G$ used to split off $B_{3}$ is tight. 
Let $u$ and $u^{\prime}$ be the vertices of the other color class of $B$ than $v_{1}$ and $v_{2}$. By Lemma 21, all the vertices of $G^{\prime}$ except possibly $u$ and $u^{\prime}$ are trimatched. Let us establish that the vertices $u$ and $u^{\prime}$ are also trimatched in $G^{\prime}$.

By symmetry, we can assume that $u$ is joined by two parallel edges to $v_{1}$. Let $u_{1}$ and $u_{2}$ be the neighbors (in $G$ ) of $u$ inside the brick $B_{1}, u_{3}$ the vertex of $B_{1}$ adjacent to $u^{\prime}$ and $u_{0}$ the remaining neighbor of $u$. Observe that $u_{0}$ is in the brick $B_{2}$. Since $B_{1}$ is trimatched, there exists a subgraph $H^{\prime}$ of $G$ spanning $B_{1}$ that contains the edges $u u_{1}, u u_{2}$ and $u^{\prime} u_{3}$ and every vertex of $B_{1}$ has degree 1 in $H^{\prime}$. Adding to $H^{\prime}$ a perfect matching of $B_{2}$ containing the edge $u u_{0}$ yields a spanning subgraph $H$ of $G^{\prime}$, in which the vertex $u$ has degree 3 and the remaining vertices have degree 1 . Since the case of the vertex $u^{\prime}$ is symmetric to that of $u$, we have proved that $G^{\prime}$ is trimatched. As argued before, the number of bricks in the brick and brace decomposition of $G$ is at most 2, i.e. $k \leq 2$.

If $k=0$, then $G=A_{2}$ which is 3 -good. If $k=1$, then $G$ is ( $\left.\geq 2\right)$-good by Lemma 16 since every brick is ( $\geq 1)$-good. If $k=2$, then $G$ is again $(\geq 2)$ good by Lemma 16 unless both $B_{1}$ and $B_{2}$ are 1-good bricks and both gluings are solo-gluings. Since the pattern of every vertex of $A_{2}$ is $(1,2,2)$, a gluing can be a solo-gluing only if the brick $B_{i}$ contains a vertex of pattern $(1,1, x)$ for some $x \in \mathbb{N}$. However, there are only three 1-good bricks containing a vertex of pattern $(1,1, x)$; see Figure 2 . In particular, both the bricks $B_{1}$ and $B_{2}$ must be one of the bricks $I_{1}, I_{2}$ and $I_{3}$.

Let us now argue that at least one of the bricks $B_{1}$ and $B_{2}$ is $I_{1}$. To this end, we prove that one of the two solo-gluings must be through a vertex of a brick with pattern $(1,1,1)$. This will yield the desired conclusion since, among $I_{1}, I_{2}$ and $I_{3}$, only $I_{1}$ contains a vertex with such a pattern. Let $G^{\prime}$ be the graph obtained from $B=A_{2}$ by solo-gluing $I_{2}$ or $I_{3}$. As argued before, the solo-gluing is through a vertex of the brick with pattern $(1,1, x)$. By the structure of $I_{2}$ and $I_{3}$, it holds that $x \geq 2$. Let $v$ be a vertex of $G^{\prime}$ that is not contained in the glued brick and $e$ an edge incident with $v$. If $e$ is contained in two different perfect matchings of $A_{2}$, then $e$ is also contained in at least two different perfect matchings of $G^{\prime}$. If $e$ is contained in a single perfect matching of $A_{2}$, then this perfect matching can be extended in $x$ different ways to the glued brick. Hence, every edge incident with $v$ is in at least two different perfect matchings of $G^{\prime}$. Since the choice of $v$ was arbitrary among the vertices not contained in the brick, we deduce that only a brick containing a vertex with pattern $(1,1,1)$ can be solo-glued to $G^{\prime}$ (recall that gluing the second brick through a vertex contained in the first one would not 

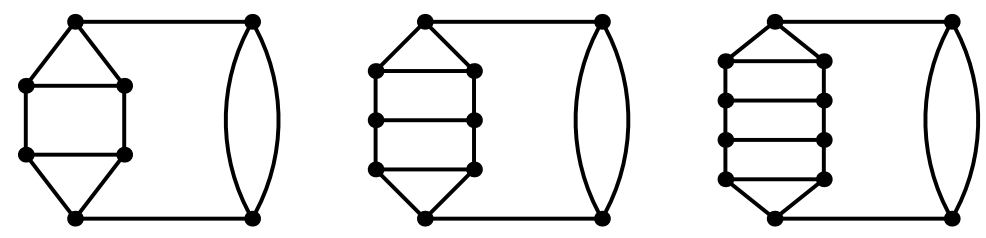

Figure 5: The graphs that can be obtained from the brace $A_{2}$ by solo-gluing the brick $I_{1}$ and one of the bricks $I_{1}, I_{2}$ and $I_{3}$ through vertices joined by parallel edges in $A_{2}$.

yield a non-trivial tight edge-cut). Hence, at least one of the bricks $B_{1}$ and $B_{2}$ is $I_{1}$.

By symmetry, we can assume henceforth that $B_{1}=I_{1}$ and $B_{2} \in\left\{I_{1}, I_{2}, I_{3}\right\}$. Let $u$ and $u^{\prime}$ be vertices of one of the color classes of $B=A_{2}$. Let $v$ be the vertex of the other color class joined by two parallel edges to $u$, and $v^{\prime}$ the vertex joined by two parallel edges to $u^{\prime}$. By symmetry, the brick $B_{1}=I_{1}$ is glued to $B=A_{2}$ through the vertex $u$. If the brick $B_{2}$ is glued through the vertex $u^{\prime}$ or the vertex $v^{\prime}$, we obtain one of the three 1-good graphs depicted in Figure 4. Note that although the brick $B_{2}$ can be glued in several nonsymmetric ways, there is a unique way to solo-glue it. Finally, if the brick $B_{2}$ is glued through the vertex $v$, then the resulting graph is $(\geq 2)$-good. See Figure 5 for the three graphs that can be obtained in this way.

It remains to analyze single-brace graphs the decomposition of which contains a brace of order at least 4 .

Lemma 25. If $G$ is a single-brace graph that contains neither $A_{2}$ nor $A_{3}$ in its brick and brace decomposition, then $G$ is $(\geq 2)$-good unless it is the graph $\mathrm{H}_{6}$ depicted in Figure 6.

Proof. Let $B$ be the brace in the decomposition of $G, n$ the order of $B$ and $B_{1}, \ldots, B_{k}$ the bricks in the decomposition. By Lemma $22, k \leq n$. Let $i_{1}$ be the number of bricks $B_{1}, \ldots, B_{k}$ isomorphic to the brick $I_{1}$. If the brace $B$ is $A_{4}$, then $B$ is 5 -good. After gluing the $i_{1}$ bricks $I_{1}$, the resulting graph $G^{\prime}$ is $\left(\geq 5-i_{1}\right)$-good by Lemma 16 . Since $G^{\prime}$ is matching double-covered by Lemma 14, none of the gluings of the other $k-i_{1}$ bricks to $G^{\prime}$ is a solo-gluing. Hence, $G$ is $\left(\geq 5-i_{1}\right)$-good. We conclude that if $G$ is not $(\geq 2)$-good, then $i_{1}=4$ and $G$ is the exceptional graph $H_{6}$ depicted in Figure 6 . 


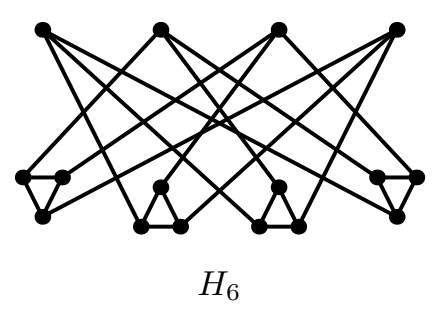

Figure 6: The exceptional graph $H_{6}$.

Assume now that $B$ is not the brace $A_{4}$. Since $B$ is also neither $A_{2}$ nor $A_{3}$ by the assumption of the lemma, $B$ is $(n+2)$-good by Lemma 19 . As in the previous paragraph, we argue that $G$ is $\left(\geq n+2-i_{1}\right)$-good. Since $i_{1} \leq k \leq n$ (the latter inequality is implied by Lemma 22), it follows that $G$ is $(\geq 2)$-good.

Lemmas 23-25 imply the following theorem. Note that every brace is $(\geq 2)$-good as shown in Section 4 .

Theorem 26. A single-brace graph $G$ is ( $\geq 2)$-good with the following exceptions:

- the graph $H_{0}$ which is 0-good, and

- the graphs $H_{1}, \ldots, H_{6}$ which are 1-good.

The exceptional graphs are depicted in Figures 3, 4 and 6.

\section{Multi-brace cubic graphs}

In this section, we analyze cubic bridgeless graphs the brick and brace decomposition of which contains at least two braces. Before we do so, we establish two auxiliary lemmas. The first one asserts that almost every single-brace graph that is not $(\geq 2)$-good is trimatched.

Lemma 27. The cubic graphs $H_{0}, \ldots, H_{6}$ are trimatched with the exception of $H_{1}$ which contains a single vertex that is not trimatched. The pattern of this vertex of $H_{1}$ is $(2,2,2)$. 

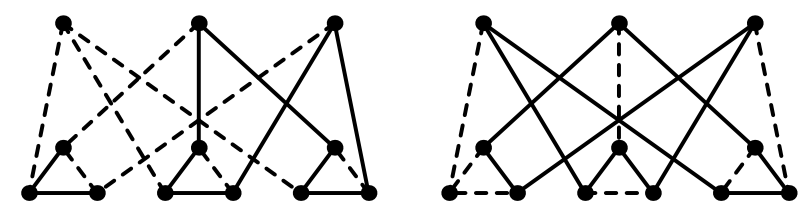

Figure 7: Spanning subgraphs of the graph $H_{0}$ showing that every vertex of $H_{0}$ is trimatched (symmetric cases are omitted). The edges contained in the subgraphs are dashed.
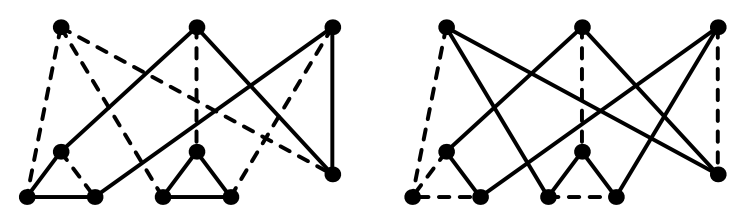

Figure 8: Spanning subgraphs of the graph $H_{1}$ showing that all but one vertices of $H_{1}$ are trimatched (symmetric cases are omitted). The edges contained in the subgraphs are dashed.
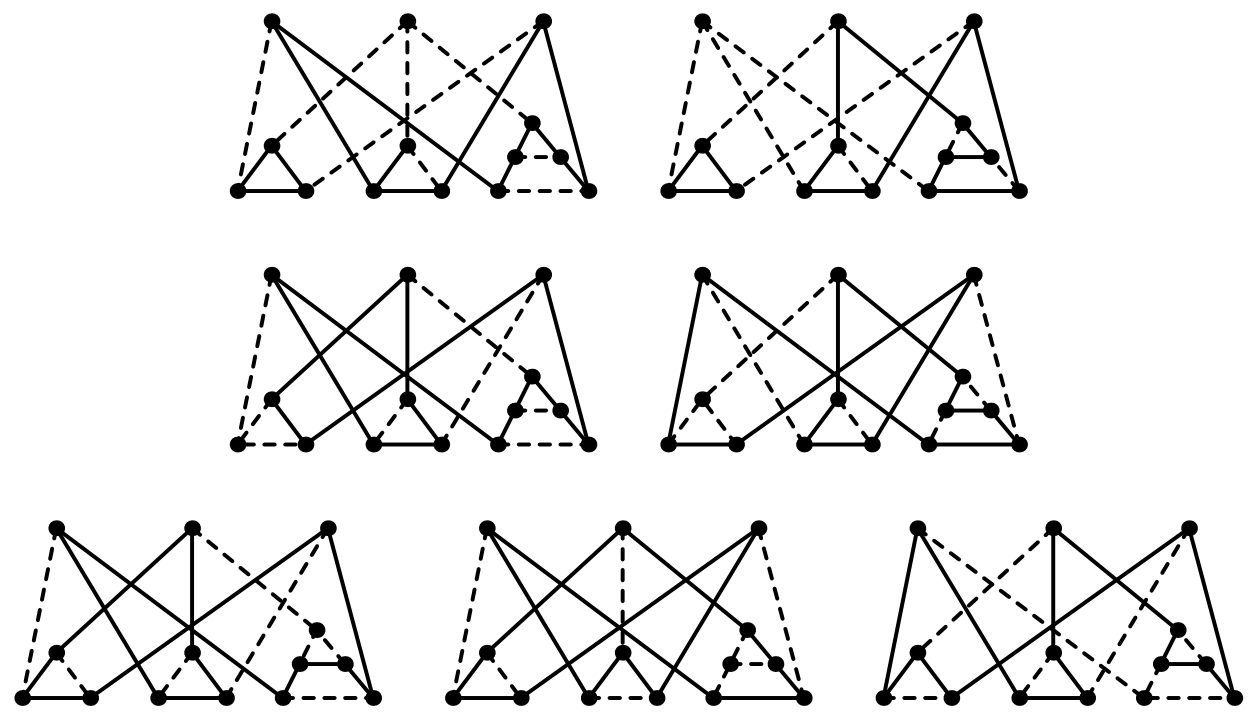

Figure 9: Spanning subgraphs of the graph $\mathrm{H}_{2}$ showing that every vertex of $\mathrm{H}_{2}$ is trimatched (symmetric cases are omitted). The edges contained in the subgraphs are dashed. 

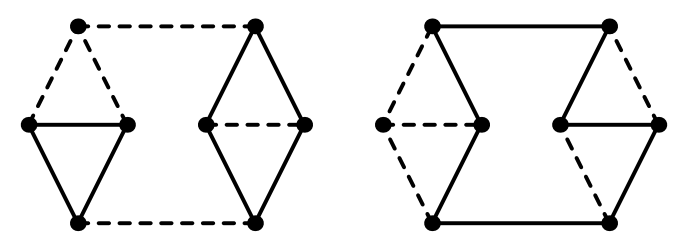

Figure 10: Spanning subgraphs of the graph $H_{3}$ showing that every vertex of $H_{3}$ is trimatched (symmetric cases are omitted). The edges contained in the subgraphs are dashed.
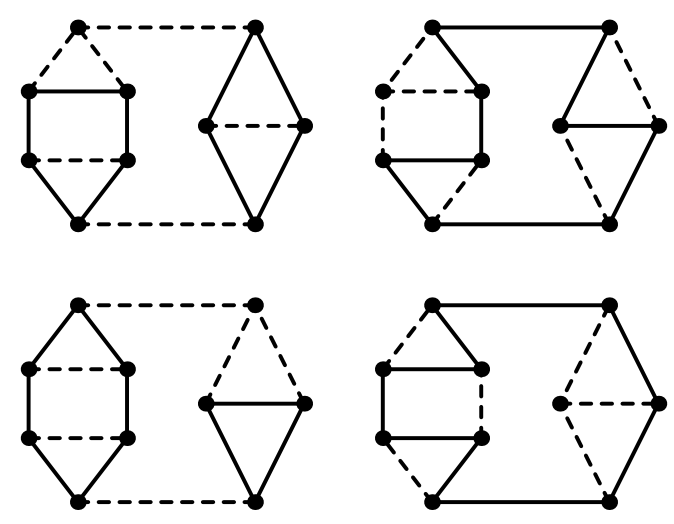

Figure 11: Spanning subgraphs of the graph $H_{4}$ showing that every vertex of $\mathrm{H}_{4}$ is trimatched (symmetric cases are omitted). The edges contained in the subgraphs are dashed. 

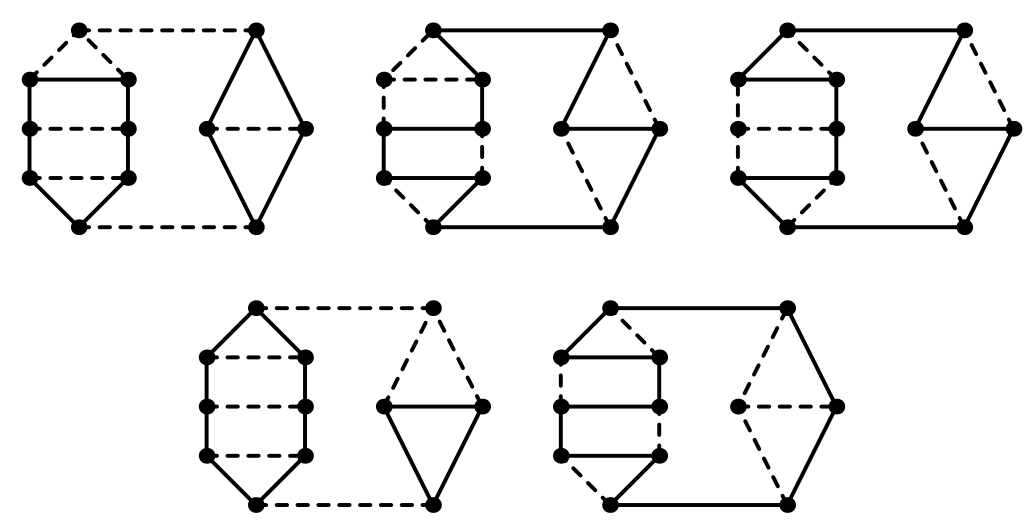

Figure 12: Spanning subgraphs of the graph $H_{5}$ showing that every vertex of $H_{5}$ is trimatched (symmetric cases are omitted). The edges contained in the subgraphs are dashed.
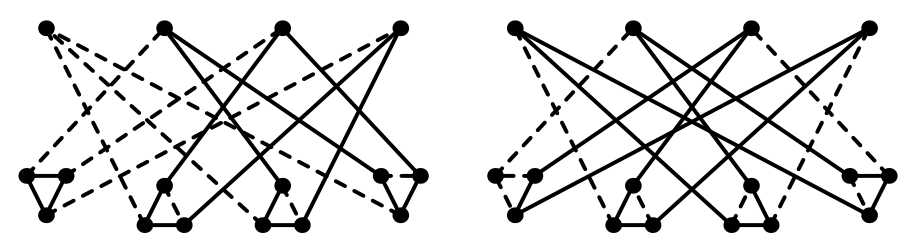

Figure 13: Spanning subgraphs of the graph $H_{6}$ showing that every vertex of $H_{6}$ is trimatched (symmetric cases are omitted). The edges contained in the subgraphs are dashed. 
Proof. It is enough to exhibit spanning subgraphs of the graphs $H_{0}, \ldots, H_{6}$ satisfying the statement of the lemma. Such subgraphs can be found in Figures 7-13; the exceptional vertex of $H_{1}$ is the vertex of $A_{3}$ of the color class where the brick $I_{1}$ was glued through the other two vertices.

In the next lemma, we restrict the structure of cubic bridgeless graphs that are not matching double-covered.

Lemma 28. If $G$ is a cubic bridgeless graph that is neither a brick nor the brace $A_{1}$, then every vertex of $G$ is incident with at most one solo-edge.

Proof. We proceed by induction on the number of vertices of $G$. If $G$ has no non-trivial tight edge-cuts, then it must be a brace. If $G$ is the brace $A_{2}$, then every vertex of $G$ has pattern $(1,2,2)$ and the statement holds. Otherwise, $G$ is matching double-covered by Lemma 14 and thus $G$ has no solo-edges at all.

Assume that $G$ has a non-trivial tight edge-cut $E=\left\{e_{1}, e_{2}, e_{3}\right\}$, and let $G_{1}$ and $G_{2}$ be the graphs obtained by splitting along $E$. Lemma 12 ensures that any non-trivial brick and brace decomposition contains at least one brace. Thus, we can assume that the brick and brace decomposition of $G_{1}$ contains a brace. By the induction hypothesis, every vertex of $G_{1}$ is incident with at most one solo-edge.

For $i \in\{1,2\}$, let $V_{i}$ be the set of vertices of $G$ contained in $G_{i}$. Further, let $v$ be the vertex of $G_{2}$ such that $G$ is obtained from $G_{2}$ by gluing $G_{1}$ through $v$. In particular, $v \notin V_{2}$. Note that the edges $e_{1}, e_{2}$ and $e_{3}$ one-toone correspond to the edges of $G_{2}$ incident with $v$. Since every vertex of $G_{1}$ is incident with at most one solo-edge, we can assume that, for each $i \in\{1,2\}$, the graph $G_{2}$ admits a perfect matching that contains the edge $e_{i}$ and can be extended to $G_{1}$ in at least two different ways.

Let $w$ be any vertex of $V_{2}$ and let $f_{1}, f_{2}$ and $f_{3}$ be the three edges incident with $w$. We aim to show that at most one of these edges is a solo-edge. Since a cubic bridgeless graph is matching covered, there exists a perfect matching $M_{1}$ of $G_{2}$ containing the edge $e_{1}$. By symmetry, we can assume that $M_{1}$ also contains the edge $f_{1}$. Since any matching containing the edge $e_{1}$ can be extended to $G_{1}$ in at least two different ways, the edge $f_{1}$ is not a solo-edge.

On the other hand, Theorem 2 implies the existence of a perfect matching $M_{2}$ of $G_{2}$ avoiding both the edges $e_{3}$ and $f_{1}$. By symmetry, we may assume that $M_{2}$ contains the edge $f_{2}$. Since $M_{2}$ also contains the edge $e_{1}$ or $e_{2}$, it can be extended to $G_{1}$ in at least two different ways. Hence, the edge $f_{2}$ is 
not a solo-edge either. We conclude that every vertex of $V_{2}$ is incident with at least two edges that are not solo-edges.

Since the number of perfect matchings containing a given edge can only increase by gluing a graph through a vertex, every vertex of $V_{1}$ is incident with at most one solo-edge. This finishes the proof of the lemma.

In the next lemma, we show that every cubic bridgeless graph $G$ that is neither a brick nor a single-brace graph contains a non-trivial tight edge-cut with a useful property.

Lemma 29. Let $G$ be a cubic bridgeless graph that is neither a brick nor a single-brace graph. Then, $G$ contains a non-trivial tight edge-cut $E$ such that neither of the graphs obtained by splitting along $E$ is a brick.

Proof. We proceed by induction on the number $K$ of graphs in the brick and brace decomposition of $G$. The result is true if $K=2$. Indeed, since $G$ is neither a brick nor a brace, Lemma 12 implies that the decomposition of $G$ contains a brace. Since $G$ is not a single-brace graph, we deduce that its decomposition is composed of two braces, which yields the sought conclusion. Assume now that $K>2$ and the theorem holds for smaller values of $K$. Since $G$ is neither a brick nor a brace, $G$ contains a non-trivial tight edge-cut $E$. Let $G_{1}$ and $G_{2}$ be the two graphs obtained from $G$ by splitting along $E$. By symmetry, we may assume that $G_{2}$ is a brick (otherwise $E$ is the sought tight edge-cut). Hence, as $G$ is not a single-brace graph, the brick and brace decomposition of $G_{1}$ contains at least two braces. Thus, by induction, $G_{1}$ contains a non-trivial tight edge-cut $E^{\prime}$ that splits $G_{1}$ into two graphs $G_{1}^{\prime}$ and $G_{2}^{\prime}$ such that neither of them is a brick, i.e., the brick and brace decomposition of both $G_{1}^{\prime}$ and $G_{2}^{\prime}$ contains a brace. Let $v$ be the vertex of $G_{1}$ such that $G$ is obtained from $G_{1}$ by gluing $G_{2}$ through $v$. By symmetry, we can assume that the vertex $v$ is contained in $G_{2}^{\prime}$.

We assert that $E^{\prime}$ is also a non-trivial tight edge-cut of $G$. Indeed, if $G$ contains a perfect matching containing all three edges of $E^{\prime}$, then this matching uses exactly one edge of $E$ because $E$ is a tight edge-cut. Hence, the edge contained in $E$ can be replaced with an edge of $G_{1}$ incident with $v$ yielding a perfect matching of $G_{1}$ containing all three edges of $E^{\prime}$.

Now split the graph $G$ along the non-trivial tight edge-cut $E^{\prime}$. One of the graphs obtained is the graph $G_{1}^{\prime}$, which is not a brick. The other graph cannot be a brick either, since its brick and brace decomposition must contain 
a brace contained in the decomposition of $G_{2}^{\prime}$ (recall that Theorem 8 ensures that the brick and brace decomposition of $G$ is unique).

We are now ready to analyze cubic bridgeless graphs the brick and brace decomposition of which contains two or more braces. We start with the case of two braces, which will be the core of our inductive argument later.

Theorem 30. If the brick and brace decomposition of a cubic bridgeless graph $G$ contains two braces, then $G$ is ( $\geq 2)$-good.

Proof. Since the brick and brace decomposition of $G$ is non-trivial, $G$ has a non-trivial tight edge-cut $E$. Let $G_{1}$ and $G_{2}$ be two graphs obtained from $G$ by splitting along $E$. By Lemma 29 , we can assume that neither $G_{1}$ nor $G_{2}$ is a brick. Hence, both $G_{1}$ and $G_{2}$ are single-brace graphs. By the definition of the brick and brace decomposition, neither $G_{1}$ nor $G_{2}$ can be the brace $A_{1}$. Note that both $G_{1}$ and $G_{2}$ have at least five perfect matchings by Lemma 20 .

Assume first that $G_{1}$ is ( $\left.\geq 2\right)$-good. By Lemma 28, the gluing of $G_{1}$ and $G_{2}$ resulting in $G$ is not a solo-gluing. Hence, if $G_{2}$ is $(\geq 1)$-good, then $G$ is $(\geq 2)$-good by Lemma 16 . If $G_{2}$ is not $(\geq 1)$-good, then $G_{2}$ must be the graph $H_{0}$ by Theorem 26. In particular, $G_{2}$ is matching double-covered. Consequently, $G$ is ( $\geq 2$ )-good by Lemma 17 since $G_{1}$ has at least five perfect matchings. A symmetric argument applies if $G_{2}$ is $(\geq 2)$-good.

It remains to consider the case where neither $G_{1}$ nor $G_{2}$ is $(\geq 2)$-good. Theorem 26 yields that each of $G_{1}$ and $G_{2}$ is one of the graphs $H_{0}, \ldots, H_{6}$. For $i \in\{1,2\}$, let $v_{i}$ be the vertex of $G_{i}$ such that $G$ is obtained from $G_{i}$ by gluing $G_{3-i}$ through $v_{i}$. At least one of the vertices $v_{1}$ and $v_{2}$ is not trimatched, since the edge-cut $E$ used to split $G$ is tight. By Lemma 27 and symmetry, we can assume that $G_{1}$ is the graph $H_{1}$ and the pattern of $v_{1}$ in $G_{1}$ is $(2,2,2)$.

If $G_{2}$ is 1 -good, then $G$ is $(\geq 2)$-good by Lemma 17 since $G_{1}$ is 1-good and matching double-covered. The other case is that $G_{2}$ is not 1-good. Then Theorem 26 implies that $G_{2}$ is the graph $H_{0}$. Consequently, the pattern of $v_{2}$ is also $(2,2,2)$, and the graph $G$ has at least $3 \cdot(2 \cdot 2)=12$ perfect matchings. Since the number of vertices of $G$ is $10+12-2=20$, the graph $G$ is 2 -good.

Finally, we can prove the main theorem of this section.

Theorem 31. If the brick and brace decomposition of a cubic bridgeless graph $G$ contains at least two braces, then $G$ is ( $\geq 2)$-good. 
Proof. The proof proceeds by induction on the number of braces in the brick and brace decomposition of $G$. If the brick and brace decomposition of $G$ contains exactly two braces, then $G$ is $(\geq 2)$-good by Theorem 30. Assume now that the decomposition of $G$ contains at least three braces. Let $G_{1}$ and $G_{2}$ be two graphs that can be obtained from $G$ by splitting along a non-trivial tight edge-cut. By Lemma 29, we can assume that neither $G_{1}$ nor $G_{2}$ is a brick. By the definition of the brick and brace decomposition, neither $G_{1}$ nor $G_{2}$ is the brace $A_{1}$.

Since the brick and brace decomposition of $G$ contains at least three braces, at least one of $G_{1}$ and $G_{2}$ is not a single-brace graph. By symmetry, we can assume that $G_{1}$ is not a single-brace graph, and thus $G_{1}$ is $(\geq 2)$-good by the induction hypothesis. The graph $G_{2}$ is $(\geq 0)$-good. This follows from Theorem 26 if $G_{2}$ is a single-brace graph, and from the induction hypothesis otherwise. By Lemma 28, the gluing of $G_{1}$ and $G_{2}$ resulting in $G$ is not a solo-gluing. So, if $G_{2}$ is ( $\left.\geq 1\right)$-good, then $G$ is $(\geq 2)$-good by Lemma 16. If $G_{2}$ is 0 -good, then $G_{2}$ must be the graph $H_{0}$ by Theorem 26 and the induction hypothesis. In particular, $G_{2}$ is matching double-covered. Moreover, $G_{1}$ has at least five perfect matchings by Lemma 20. Hence, Lemma 17 implies that $G$ is $(\geq 2)$-good.

Theorems 15, 26 and 31 imply Theorem 7, the main result of this paper.

\section{Acknowledgment}

This research was done while the first two authors were visiting the third at Technische Universität Ilmenau. They thank their host for providing a perfect working environment.

\section{References}

[1] de Carvalho, M. H., Lucchesi, C. L., and Murty, U. S. R. Graphs with independent perfect matchings. J. Graph Theory, 48(1):19-50, 2005.

[2] Chudnovsky, M. and Seymour, P. Perfect matchings in planar cubic graphs, 2008. Submitted for publication.

[3] Edmonds, J., Lovász, L., and Pulleyblank, W. R. Brick decompositions and the matching rank of graphs. Combinatorica, 2(3):247-274, 1982. 
[4] Esperet, L., Kardoš, F., and Král', D. A superlinear bound on the number of perfect matchings in cubic bridgeless graphs. In preparation.

[5] Esperet, L., Král', D., Škoda, P., and Škrekovski, R. An improved linear bound on the number of perfect matchings in cubic graphs. Submitted for publication.

[6] Gurvits, L. Hyperbolic polynomials approach to Van der Waerden/Schrijver-Valiant like conjectures: sharper bounds, simpler proofs and algorithmic applications. In STOC'06: Proceedings of the 38th Annual ACM Symposium on Theory of Computing, pages 417-426. ACM, New York, 2006.

[7] Hartfiel, D. J. and Crosby, J. W. On the permanent of a certain class of (0, 1)-matrices. Canad. Math. Bull., 14:507-511, 1971.

[8] Lovász, L. Matching structure and the matching lattice. J. Combin. Theory Ser. B, 43(2):187-222, 1987.

[9] Lovász, L. and Plummer, M. D. Matching theory, Volume 121 of NorthHolland Mathematics Studies. North-Holland Publishing Co., Amsterdam, 1986. Annals of Discrete Mathematics, 29.

[10] Minc, H. On lower bounds for permanents of $(0,1)$ matrices. Proc. Amer. Math. Soc., 22:117-123, 1969.

[11] Naddef, D. Rank of maximum matchings in a graph. Math. Programming, 22(1):52-70, 1982.

[12] Petersen, J. Die Theorie der regulären graphs. Acta Math., 15(1):193220, 1891.

[13] Plesník, J. Connectivity of regular graphs and the existence of 1-factors. Mat. Časopis Sloven. Akad. Vied, 22:310-318, 1972.

[14] Schrijver, A. Counting 1-factors in regular bipartite graphs. J. Combin. Theory Ser. B, 72(1):122-135, 1998.

[15] Schrijver, A. Combinatorial optimization. Polyhedra and efficiency. Vol. A, Volume 24 of Algorithms and Combinatorics. Springer-Verlag, Berlin, 2003. Paths, flows, matchings, Chapters 1-38. 
[16] Schrijver, A. and Valiant, W. G. On lower bounds for permanents. Nederl. Akad. Wetensch. Indag. Math., 42(4):425-427, 1980.

[17] Sinkhorn, R. Concerning a conjecture of Marshall Hall. Proc. Amer. Math. Soc., 21:197-201, 1969.

[18] Voorhoeve, M. A lower bound for the permanents of certain $(0,1)$ matrices. Nederl. Akad. Wetensch. Indag. Math., 41(1):83-86, 1979. 